\title{
Telehealth and autism: A systematic search and review of
}

\author{
the literature
}

Rebecca Sutherland, David Trembath \& Jacqueline Roberts

To cite this article: Rebecca Sutherland, David Trembath \& Jacqueline Roberts (2018)

Telehealth and autism: A systematic search and review of the literature, International Journal of Speech- Language Pathology, 20:3, 324-336, DOI: 10.1080/17549507.2018.1465123

To link to this article: https://doi.org/10.1080/17549507.2018.1465123

Published online: 30 Apr 2018. 


\begin{abstract}
Purpose: Research interest in telehealth and autism spectrum disorder (ASD) has grown. There is a need to review the literature to allow speech-language pathologists (SLPs) and other service providers to consider applicability to their settings. The aim of this review was to examine the nature and outcomes of studies examining telehealth assessment and/or intervention in ASD.
\end{abstract}

Method: A systematic search of the literature was undertaken, with 14 studies meeting inclusion criteria. The authors extracted information from each included article, including participant characteristics, technology used, measures and reported outcomes. Quality review of articles was undertaken.

Result: The 284 participants with ASD across the 14 included studies ranged in age from 19 months to adulthood. The quality of the studies varied. A range of services were provided via telehealth, including diagnostic assessments, early intervention and language therapy. Results suggested that services delivered via telehealth were equivalent to services delivered face to face, and superior to comparison groups without telehealth sessions.

Conclusion: The findings suggest there may be a range of benefits in using telehealth with individuals with ASD, their families, and teachers. Further research, however, is required particularly regarding the use of telehealth directly with children with ASD for assessment and intervention.

Keywords: autism spectrum disorder, telehealth, speech-language pathology 


\section{Introduction}

Telehealth is a broad term describing health care services that utilise information communication technology to deliver services. In the speech-language pathology context, telehealth has been defined as the "the application of telecommunications technology to deliver professional services at a distance by linking clinician to client, or clinician to clinician for assessment, intervention, and/or consultation" (American Speech and Hearing Association, n.d.p.1). These approaches have evolved from the early use of audio and video tapes and telephone discussions (e.g. Harrison, Wilson, \& Onslow, 1999), to the current use of both sophisticated real time communication technology over the internet, with data and image sharing using equipment ranging from custom built videoconferencing suites, as well as low cost internet based communication software (e.g. Skype) with headsets, webcams, speakers, handheld cameras, microphones, and internet connectivity (Keck \& Doarn, 2014). Telehealth in speech-language pathology is becoming an established practice in certain areas, with research indicating successful use in treating fluency disorders (Harrison et al., 1999; Lewis, Packman, Onslow, Simpson, \& Jones, 2008; O’Brian, Smith, \& Onslow, 2014; Wilson, Onslow, \& Lincoln, 2004), dysphagia (Sharma, Ward, Burns, Theodoros, \& Russell, 2013; Ward, Burns, Theodoros, \& Russell, 2013), adult speech and language disorders (Constantinescu et al., 2010; McGrath, Dowds, \& Goldstein, 2008) and speech sound disorders in childhood (Grogan-Johnson et al., 2013). Telehealth has also been used successfully in formal language assessments in children (Sutherland et al., 2017; Waite, Theodoros, Russell, \& Cahill, 2010). Based on these successes, clinicians and researchers are beginning to explore the potential for telehealth as a tool for assessment and intervention across a range of practice areas, including working with individuals with autism spectrum disorder (ASD). 


\section{Telehealth Applications in Autism Spectrum Disorder (ASD)}

Autism spectrum disorder (ASD) is a neurodevelopmental disability characterised by differences in social communication and social interactions, as well as restricted, repetitive patterns of behaviour, interests, or activities (American Psychiatric Association, 2013). It is a lifelong condition, meaning that individuals and their families are likely to require a range of interventions over a long period of time. A number of evidence-based programs exist, including Early Intensive Behavioural Intervention (Prior, Roberts, Rodgers, Williams \& Sutherland, 2011) and the Early Start Denver Model (Dawson et al., 2010), but access to these programs, and/or training clinicians or parents to administer these programs in the community is challenging. For families who live remotely from services, and those who are limited by transport and other logistical difficulties, accessing appropriate services in a timely way can be difficult and telehealth may be a useful primary or adjunct intervention mode. Despite the success of telehealth in other areas, the literature regarding telehealth use with students with diverse learning needs, such as ASD to deliver assessment and intervention is limited.

Prior to the current review, Boisvert, Lang, Andrianopoulos \& Boscardin (2010) completed the most recent systematic review of research related to autism and telehealth, which yielded only eight studies that met their inclusion criteria: the study involved at least one person with ASD, the use of telehealth between an expert clinician and a remote site and at least one dependent measure involving the individual with ASD and the results of an assessment or evaluation of changes in behaviour. There were 46 participants involved across the eight studies (range $n=1-29$ ) with an age range of $2-11$ years. The services provided included behaviour analysis, teacher, therapist or parent training in particular techniques or interventions, psychiatric consultations and ongoing consultations regarding individual education plans. The overall results of the review were positive, in terms of the successful 
implementation of the telehealth services, reduced challenging behaviours, improved socialcommunication, and participant satisfaction across the various studies. However, it was noted that a minority of studies employed an experimental design and some studies reported significant technical difficulties. Furthermore, none of the included studies involved direct telehealth services to the individual with ASD.

Research interest in telehealth as an adjunct or equivalent practice to face to face services has grown since the last systematic review, as evidenced by the growth in publications. For instance, a search using the PsycINFO database using the search terms ‘telehealth OR telepractice” revealed 387 articles published between 2000 and 2010, with 619 articles in the five years to 2016. As a result, there is a need to review the literature regarding the use of telehealth to allow individual SLPs and service providers more broadly to consider applicability to their settings. Accordingly, the aim of this review is to document the research into the use of telehealth for autism spectrum disorder since the previous (2010) systematic review, with regards to a) the type of service delivered, b) the recipients of the telehealth service, and c) the outcomes of the telehealth services provided.

\section{Method}

The Preferred Reporting Items for Systematic Reviews and Meta-Analyses (PRISMA) statement was used to guide the systematic review process (see Figure 1); the protocol for this review was not published a priori. A systematic search of the literature was undertaken using the databases Medline, PsycINFO, ERIC, CINAHL and PUBMED for articles published up to December 2016. These databases are in keeping with the databases searched in the previous review by Boisvert et al. (2010). Titles and abstracts were searched using key words to describe telehealth and ASD ((“telehealth" OR "telemedicine" OR "telepractice" OR “telecare”) AND (“Autis* (allowing searches for Autism, Autistic, Autisms etc.)” OR 
“Asperger” OR “PDD-NOS” (Pervasive Developmental Disorder - Not Otherwise

Specified)). The search was limited to English language. In keeping with the previous systematic review, only articles appearing in peer reviewed journals were included. There was no limit on date of publication, however, no eligible articles were found prior to 2010 that were not included in the previous review.

A total of 170 articles that included both the telehealth and autism search terms were found across the five databases. After duplicates were removed, 86 remained. Titles and abstracts were screened by the first author based on predetermined inclusion criteria including: (a) inclusion of at least one individual with autism or parent of a person with autism; (b) implementation of a telehealth system for the purpose of assessment or intervention; (c) utilisation of a design that allows for experimental control (in the case of intervention studies) or comparison condition (in the case of diagnostic studies); (d) measurement of factors associated with telehealth implementation (such as child outcomes, feasibility, parent outcomes); and (e) published in a peer reviewed journal. Both synchronous (real time consultation) and asynchronous (capturing digital samples such as images or videos in one location and transmitting these to a remote clinician for consultation or interpretation (Deshpande, et al., 2009) telehealth modalities were considered. However, studies that described parent, teacher or clinician training using web-based materials but without consultation were not included as these were not considered telehealth in this context, as per the ASHA (2017) definition of telehealth (e.g. Fisher et al., 2014). General reviews or discussion articles (e.g. Goldstein \& Myers, 2014; Pearl et al., 2014; Reese et al., 2015; Savin, Garry, Zuccaro, \& Novins, 2006; Terry, 2009) were excluded as were articles describing other technology such as wearable sensors (Fletcher, Poh, \& Eydgahi, 2010), virtual reality (Max \& Burke, 1996) or technology in general (Aresti-Bartolome \& GarciaZapirain, 2014). The eight studies included in the Boisvert et al. (2010) systematic review 
were not included; these been described adequately in the previous systematic review. There were no studies published before 2010 that were not included in the prior review. After screening the 86 papers based on title and abstract, 40 papers remained. These were independently read in full by two reviewers (the first and third authors) in order to determine eligibility with discussion to resolve differences. Following review, 14 papers remained that met all criteria. A hand search of the references of these 14 papers was completed with eight additional articles identified by their titles as being of potential interest; of these, four did not involve any participants with ASD (Boydell, Volpe, \& Pignatiello, 2010; Cefai, Smith, \& Pushak, 2010; Gros et al., 2013; Taylor et al., 2008) and the remaining four involved online learning, eLearning or computer based training modules without an interactive synchronous or asynchronous telehealth component (Buzhardt \& Heitzman-Powell, 2005; Hamad, Serna, Morrison, \& Fleming, 2010; Jang et al., 2012; Wainer \& Ingersoll, 2013), leaving the number of included articles at 14.

Quality review of included intervention articles was undertaken using the Scientific Merit Rating Scales (SMRS; National Autism Center, 2015). The process involved rating the intervention research studies on five dimensions of experimental rigour (research design, participant ascertainment, generalisation, and measurement of dependent and independent variables). These ratings are then combined and an overall scientific merit score obtained. Scores of 3, 4, or 5 indicate that sufficient scientific rigor has been applied, a score of 2 indicates initial evidence about treatment effects and a score of 0-1 indicates that insufficient scientific rigour has been applied to the population of individuals with ASD. The first and third authors extracted the following information from each included article: participant characteristics (age, diagnosis, gender), the technology used, the type of service, the study design/methodology, measures, and reported outcomes.

(Figure 1 about here) 


\section{Result}

The details of the included papers are summarised in Table I. Fourteen papers, all originating from the United States, were published between 2010 and 2016, across a range of disciplines as described below.

\section{(Table I about here)}

\section{Participant Characteristics}

Participants with ASD. Participants with ASD were directly and actively involved in telehealth (that is, interacting with a clinician via a telehealth platform) in three studies: twenty-three adults were participants in one study that investigated remote diagnosis (Schutte et al., 2015); a child aged 11 years was engaged in language intervention (Boisvert, Hall, Andrianopoulos, \& Chaclas, 2012), and; 17 students with a mean age of 11 years were involved with their parents in an anxiety program (Hepburn, Blakeley-Smith, Wolff, \& Reaven, 2015). A further 243 children with ASD were involved in the studies via their parents' or teachers' interaction with telehealth and child outcomes of interventions measured. Ages of the children ranged from 19 months to early adolescence.

Parents. Multiple studies investigated telehealth interventions aimed at parents with the total number of parents, across all studies, equalling 211 . The age of the children ranged from 19 months to early adolescence.

Professionals. Forty-nine teachers were participants in one study (Ruble et al.,2013) as part of teacher/child dyads.

\section{Technology}

The majority of the studies utilised off-the-shelf, readily available equipment, including desk top computers, lap tops, webcams and tablet computers. One study used a 
specially designed platform (VISYTER; Schutte et al., 2015). Publicly available videoconferencing software such as Skype, Oovoo and Adobe Connect was used in 10 of the studies; three studies used unspecified password protected videoconferencing systems.

\section{Service type}

The studies investigated a diverse range of service types, as described below:

Diagnostic assessment. Three papers examined telehealth diagnostic services. Reese et al. (2013) and Reese et al. (2015) used video conferencing between rooms in the same building to observe parents administering parts of the Autism Diagnostic Observation Schedule - 2 (ADOS-2), a play-based diagnostic assessment. Parents were randomly assigned to a video conferencing condition (VC) or an in-person condition (IP). In both studies, parents watched a video demonstrating administration of parts of the ADOS-2, received information and were then coached through elements of the assessment by trained observers. Parents in the VC condition received coaching via clinicians using the video conferencing system; parents in the IP condition received coaching by a clinician in the same room. Schutte et al. (2015), in contrast, used a specially designed platform (known as VISYTER) to deliver Module 4 of the ADOS-2 to 23 adults with known diagnoses of autism spectrum disorder.

Early intervention. Six studies examined telehealth and early intervention. Early intervention services delivered via telehealth included 'Program ImPACT' (Ingersoll \& Berger, 2015; Ingersoll, Wainer, Berger, Pickard, Bonter, 2016; Pickard, Wainer, Bailey \& Ingersoll; 2016), 'iPICS' (Meadan, et al., 2016), a general communication intervention (Baharav \& Reiser, 2010) and an imitation training (Wainer \& Ingersoll, 2014). All the interventions were delivered via telehealth to parents. Five of the studies compared an online training only condition (non-telehealth condition) with online training plus parent telehealth 
sessions (telehealth condition); the sixth study (Baharav \& Reiser, 2010) used a singlesubject time-series (A-B) repeated measures design to compare telehealth sessions to intervention as usual (that is, face to face sessions).

Anxiety intervention. One study (Hepburn et al., 2015) delivered an anxiety intervention ('Telehealth Facing Your Fears (FYF) Intervention') to 17 parent-child dyads via telehealth. Child participants had an average age of 11.5 years and all were diagnosed with both autism and anxiety. Outcomes were compared to a waiting list cohort $(n=16)$.

Functional behaviour assessment and functional communication training. Two studies of functional assessment of behaviour and functional communication training were included in the review. Lindgren et al. (2016) compared the outcomes of 71 parents of children with autism and/or developmental delays who were trained to deliver functional assessment and functional communication training in three different conditions: (1) in home, face-to-face parent training; (2) coaching via video conferencing at a regional centre; and (3) in-home coaching via telehealth. Suess et al. (2014) examined functional analysis and communication training for three parents, comparing coached (via telehealth) and independent trials.

School age-intervention. Ruble et al. (2013) provided intervention to 49 teacherchild dyads aged $3-9$ in three groups: (1) web-based education with face to face consultation; (2) web-based education with web-based consultation; and (3) a group that received web-based education only (non-telehealth). The goals were individualised and not described in detail in the article.

Language intervention. One study (Boisvert, et al., 2012) evaluated the impact of language intervention delivered via telehealth to an 11-year-old boy on the autism spectrum. 
The focus of the intervention was the use of transition words in narratives, with conventional intervention compared with telehealth intervention.

\section{Study Design/Method}

A range of methodologies was employed across the 14 studies. Five studies utilised a single subject, changing conditions design with repeated measures (Baharav \& Reiser, 2010; Boisvert et al., 2012; Meadan et al., 2016; Suess et al., 2014; Wainer \& Ingersoll, 2014). Two studies employed a cohort comparison methodology comparing the telehealth intervention groups with a non-treatment group from another study (Hepburn et al., 2016) and with previous treatment groups using face to face and centre based telehealth interventions (Lindgren et al., 2016). Four intervention studies compared intervention groups utilising a randomised control trial design (Ingersoll \& Berger, 2015; Ingersoll et al., 2016; Pickard et al., 2016; Ruble et al., 2013) with two diagnosis studies using randomisation to allocate participants to assessment conditions (Reese et al., 2013; Reese et al., 2015). A third diagnosis study employed a within subjects crossover design to compare telehealth and face to face assessment outcomes (Shutte et al., 2015). With respect to comparison conditions, six studies compared online learning plus telehealth sessions to online learning alone (nontelehealth) (Baharav \& Reiser, 2010; Ingersoll \& Berger, 2015; Ingersoll et al., 2016; Meadan, et al., 2016; Pickard et al., 2016; Wainer \& Ingersoll, 2014), six studies compared telehealth with face to face conditions (Boisvert et al., 2012; Lindgren et al. 2016; Reese et al., 2013; Reese et al., 2015; Ruble, et al., 2013; Shutte et al., 2015), and two studies compared telehealth with no intervention or intervention as usual (Hepburn et al., 2016; Ruble et al., 2013).

The quality of the studies based on the SMRS (National Autism Center, 2015) varied. One case study involving a single participant (Boisvert et al., 2012) utilised a repeated 
measures design with multiple probes but the small number of participants and lack of generalisation measures meant that it scored <2 (indicating insufficient scientific rigour). Three studies scored between 2.0 - 2.9 (initial evidence; Baharav \& Reiser; 2010; Lindgren et al., 2016; Ingersoll \& Berger, 2015) and the remainder of the studies were scored 3 or 4 (sufficient scientific rigour). The three studies regarding diagnosis (Reese et al., 2013; Reese et al., 2014; Schutte et al., 2015) were not suitable to be rated by the SMRS system but were considered adequate in terms of participant ascertainment, sample size, data collected, and fidelity measures.

\section{Clinical Outcomes}

A range of participant, parent and teacher outcomes were measured across the included studies. These have been categorised as follows for descriptive purposes.

Diagnosis. Reese et al. (2013) and Reese et al. (2015) compared the outcomes of diagnostic assessment delivered face to face, with the results when delivered via telehealth and found high levels of agreement in diagnostic status between the conditions. Greater discrepancies in agreement on individual items was noted. Similarly, Shutte et al. (2015) reported high levels of agreement regarding diagnostic categories on the ADOS (i.e. Autism, Autism Spectrum Disorder or not ASD) but with variation in the reliability of individual items.

Communication. Eight studies reported on communication outcomes (Baharav \& Reiser, 2010; Boisvert, et al., 2012; Ingersoll, et al., 2016; Meadan, et al., 2016; Pickard, et al., 2016; Ruble, et al., 2013; Wainer \& Ingersoll, 2014). Positive impacts on communication, including improved initiations and responses, as measured through formal and observation measures were reported by Baharav \& Reiser (2010). Similarly, the Boisvert et al. (2012) study reported better use of transition words after telehealth therapy compared with baseline 
and that the students' performance was more consistent in telehealth condition compared with face to face condition. Ingersoll et al. (2016) used structured observations as well as standardised questionnaires and found that both groups (self-directed online learning and therapist assisted online learning) showed improvements, with the therapist assisted group showing significant increases in their standard scores on the social domain of the Vineland Adaptive Behaviour Scales $-2^{\text {nd }}$ edition while children in the self-directed condition did not. Pickard et al. (2016), in their article about the same study as described by Ingersoll et al. (2016), reported that parents in the therapist assisted group were more likely to report gains in social communication than the self-directed condition parents. Ruble et al. (2013) used 'Psychometrically Equivalence Tested Goal Attainment Scaling (PET-GAS)' to measure outcomes across a wide range of individual communication, social and learning goals for 49 teacher-child dyads. Results showed similar mean PET-GAS change when comparing the face to face coaching with telehealth coaching and that both groups were better than the noncoached group. The communication outcomes were less robust for the three children in the Meaden et al. (2016) with the authors suggesting there were "no clear results across dyads for children's communication behaviour in the multiple-baseline analysis" (p.17). Wainer \& Ingersoll (2014) reported similarly variable results for rates of imitation in their study comparing baseline with self-directed (non-telehealth) and coaching conditions.

Behaviour. Lindgren et al. (2016) used functional analysis (FA) and functional communication training (FCT) to reduce challenging behaviour, and found that the mean percentage reduction in problem behaviour was more than $90 \%$ for the three groups (home based, centre based telehealth and home-based telehealth). Suess et al. (2014) also reported on a reduction in problem behaviours following telehealth training and coaching.

Anxiety. The anxiety study (Hepburn et al., 2015) that delivered an intervention to 17 parent-child dyads found statistically significant differences between the experimental and 
comparison group (wait list) on the anxiety measures using Screen for Anxiety and Related Emotional Disorders in Children (SCARED; Birmaher et al., 1999).

\section{Implementation outcomes}

Reduced costs. One study (Lindgren et al., 2016) looked specifically at the costs associated with home based telehealth compared with face to face sessions and centre based telehealth, and found that overall costs were lowest for the home telehealth group.

Satisfaction and acceptability. Parent satisfaction was a reported outcome for nine of the 14 studies (Baharav \& Reiser, 2010; Hepburn et al., 2016; Ingersoll \& Berger, 2015; Meadan, et al., 2016; Pickard, Wainer, Bailey \& Ingersoll, 2016; Reese et al., 2015; Schutte et al., 2015; Suess et al., 2014; Wainer \& Ingersoll, 2014). All studies reported high levels of program acceptability and parent satisfaction with the telehealth component of the intervention or assessment. In addition, two studies that involved direct telehealth involvement with individuals on the spectrum (Hepburn, Blakeley-Smith, Wolff \& Reaven, 2016; Schutte et al., 2015) reported high participant satisfaction with the methods used.

Fidelity. Measurement of fidelity of parent implementation of tasks was a focus of seven of the 14 studies (Hepburn, et al., 2016; Ingersoll \& Berger, 2015; Ingersoll, et al., 2016; Meadan, et al., 2016; Reese et al., 2013; Suess et al., 2014; Wainer \& Ingersoll, 2014). The fidelity of parent administration of assessment tasks in the diagnosis study (Reese et al., 2013) was described as 'adequate', while parent fidelity in the behaviour study of Suess et al. (2014) varied across the coached and independent trials. The remaining studies reported high levels of parent fidelity for interventions provided via telehealth (Hepburn et al., 2016; Ingersoll et al., 2016), with a number reporting that the fidelity of programs taught to parents online was improved when telehealth coaching was provided (Ingersoll \& Berger, 2015; Meadan, et al., 2016; Wainer \& Ingersoll, 2014). 


\section{Discussion}

The present review sought to examine the literature regarding telehealth and autism published since the previous systematic review (Boisvert et al., 2010) with regards to the type of service delivered, the recipients of the telehealth service, and the outcomes of the telehealth services provided. The 284 participants on the autism spectrum across the 14 included studies ranged in age from 19 months to adulthood. A range of services were provided by telehealth, including diagnostic assessments, early intervention, functional behavioural analysis, and anxiety interventions. One study addressed language impairment directly. Outcomes were largely positive with the results suggesting that services delivered via telehealth were equivalent to services delivered face to face, and superior to comparison or control groups (who did not receive the intervention), or self-directed learning without telehealth sessions.

There has been a considerable increase in research interest in this area since the previous systematic review (Boisvert et al., 2010) which found only eight eligible studies, representing just 46 participants and with only a minority of the studies employing an experimental design. Accordingly, we were able to extend the findings of the previous review, not only in terms of time frame, but through examination of the outcomes of telehealth compared with non-telehealth services (including service delivery as normal, online learning only, or face-to-face interactions). To this end, a criterion for inclusion was that the study involved a comparison condition. The 14 studies that satisfied this criterion, featured a range of methodologies, including single case study design as well as more rigorous randomised control trials. It was noted that such studies were still in the minority of the 40 studies screened, with 26 studies that met the other inclusion criteria but that did not include a comparison condition. As telehealth becomes increasingly accepted and widely used, it is essential that research examining its use features designs that allow for robust examination of its equivalence, or otherwise, with practice as usual. 
In considering the relevance of the findings to speech-language pathology practice, it is important to note that most included studies focused on telehealth for young children and their parents, carers, and/or teachers. In addition, the vast majority of the recipients of telehealth interventions (that is, the person interacting with the therapist online) were parents, carers or teachers; of the 284 participants, only 23 adults and 18 older children on the autism spectrum were directly engaged in the telehealth technologies. Only one child was directly involved in telehealth without involvement in a parent dyad (Boisvert et al., 2012) and as such, outcomes related to the provision of telehealth services to individuals with ASD are difficult to generalise. This represents a significant gap in the literature with very little yet known about the reactions and behaviours of children and adolescents on the autism spectrum to telehealth in comparison with face-to-face conditions.

A number of limitations of the included studies were noted. Small sample sizes continue to limit the generalisability of findings in some studies, with five of the studies involving five or fewer participants $(n=1$ to $n=5)$. It is positive to see the growth in the inclusion of comparison groups and/or conditions since the last review. However, group research continues to be limited by lack of randomisation and comparison with non-matched groups in some studies (e.g. Lindgren et al., 2016). The use of changing conditions design with repeated measures (e.g. Baharav \& Reiser, 2010; Boisvert et al., 2012; Meadan et al., 2016; Suess et al., 2014; Wainer \& Ingersoll, 2014), while providing a comparison between two conditions, is also limited by potential sequence effects (e.g. Meaden et al., 2016). Only three studies (Ingersoll, et al., 2016; Meadan, et al.,2016; Wainer \& Ingersoll, 2015) included measures of outcome maintenance or generalisation.

The use of telehealth to provide diagnostic assessments for autism was explored by three studies in this review. Two of these studies (Reese et al., 2013; Reese et al., 2015) used parents to deliver items of the Autism Diagnostic Observation Schedule (ADOS; Lord et al., 
2012) to their children. While the authors reported good agreement on diagnostic status when in-person and telehealth observations were compared (Reese et al., 2013), as well as good accuracy when telehealth assessment outcomes were compared with later assessments (Reese et al., 2015) along with adequate parent fidelity, it is unclear to what extent the impact of the parent implementation of the assessments may have had. Parents were given information about the activities prior to the assessments and then received coaching during the assessment to implement the items which is a significant departure from standard practice. As initial studies, neither trialled providing a full autism diagnostic assessment with feedback to parents and both studies were conducted via a telehealth simulation (rooms connected by closed circuit television in the same building), suggesting that the use of telehealth for diagnostic assessments should not yet be considered an established practice. In addition, the studies used percentage agreement to measure agreement between the assessment methods, rather than a more rigorous measure of agreement appropriate for method comparison studies, such as Bland-Altman analysis (Bland \& Altman, 1999; Taylor, Armfield, Dodrill \& Smith, 2014).

\section{Limitations}

The current review was limited by the small number of included studies and a metaanalysis of outcomes was not completed in the current study. Despite the growth in research and apparent uptake in the use of telehealth, only 14 studies met the inclusion criteria for this review. As a result, the findings of this study need to be interpreted with caution. In addition, there was a wide range of service types assessed in the included studies, meaning that the data on any one area are limited. Similarly, a lack of consistency, common measurement tools and clear reporting of outcomes makes completing meta-analysis impossible. This review was also limited by the lack of inclusion of grey literature and unpublished data. In addition, the first phase of screening articles in this study was completed by the first author 
only. Although subsequent phases of the review were completed by two of the authors, reliability of data extraction was not conducted. In addition, it is possible that appropriate articles may have been missed in this process. All included studies originated from one country (United States), potentially limiting generalisation of the findings.

\section{Future directions}

The results of this review suggest that telehealth continues to be a promising area of practice for individuals on the autism spectrum. Studies of telehealth in the past six years have focused strongly on the provision of telehealth instruction to parents, caregivers, and teachers across a range of service types and utilising a number of study designs. There are, however, gaps in the research that future studies could consider. The first of these is exploration of the role of telehealth in providing direct services to people with ASD. Few studies have examined the reactions and behaviours of children and young people on the autism spectrum when provided with telehealth services, with only one single-subject study focused on language intervention provided via telehealth directly to a child on the spectrum. Research that assists speech-language pathologists to make decisions about providing telehealth services to children on the spectrum, such as research that considers the reactions and behaviours of these children when interacting with therapists would be valuable. Future studies also need to continue to include comparison conditions to ensure that clinicians, parents and administrators can be confident that telehealth services are at least equivalent to services provided via intervention as usual. Finally, while the studies included in the current review reported high rates of participant satisfaction, future studies should continue to explore the facilitators and barriers to the use of telehealth with clinicians, parents and individuals on the autism spectrum.

\section{Conclusion}


Telehealth has been shown to have great potential in the assessment and intervention for a range of conditions relevant to speech-language pathologists. There is growing research into the types of services offered, particularly for individuals with ASD, and there is evidence that the design and methodology of the research conducted is becoming more rigorous. This review suggests there may be a range of benefits in using telehealth to provide assessment, training and direct intervention using telehealth to individuals with ASD, their families and teachers. Further research, however, is required particularly regarding the use of telehealth directly with children with ASD for speech and language interventions.

\section{Author Declaration}

The authors have no conflict of interest to report. 


\section{References}

American Psychiatric Association. (2013). Diagnostic and statistical manual of mental disorders (5th ed.). Washington, D.C: American Psychiatric Association.

American Speech and Hearing Association. (n.d.). Telepractice. Retrieved from http://www.asha.org/Practice-Portal/Professional-Issues/Telepractice/

Aresti-Bartolome, N., \& Garcia-Zapirain, B. (2014). Technologies as support tools for persons with autistic spectrum disorder: a systematic review. International Journal of Environmental Research and Public Health, 11(8), 7767-7802.

Baharav, E., \& Reiser, C. (2010). Using telepractice in parent training in early autism. Telemedicine and e-Health, 16(6), 727-731. 10.1089/tmj.2010.0029

Birmaher, B., Brent, D. A., Chiappetta, L., Bridge, J., Monga, S., \& Baugher, M. (1999). Psychometric properties of the Screen for Child Anxiety Related Emotional Disorders (SCARED): a replication study. Journal of the American Academy of Child \& Adolescent Psychiatry, 38(10), 1230-1236. doi:https://doi.org/10.1097/00004583199910000-00011

Bland, J. M., \& Altman, D. G. (1999). Measuring agreement in method comparison studies. Statistical Methods in Medical Research, 8(2), 135-160. doi:10.1177/096228029900800204

Boisvert, M., Lang, R., Andrianopoulos, M., \& Boscardin, M. L. (2010). Telepractice in the assessment and treatment of individuals with autism spectrum disorders: A systematic review. Developmental Neurorehabilitation, 13(6), 423-432. $10.3109 / 17518423.2010 .499889$

Boisvert, M., Hall, N., Andrianopoulos, M., \& Chaclas, J. (2012). The multi-faceted implementation of telepractice to service individuals with autism. International Journal of Telerehabilitation, 4(2), 11-24. doi:http://dx.doi.org/10.5195/ijt.2012.6104 
Boydell, K. M., Volpe, T., \& Pignatiello, A. (2010). A qualitative study of young people's perspectives on receiving psychiatric services via televideo. Journal of the Canadian Academy of Child and Adolescent Psychiatry 19(1), 5.

Buzhardt, J., \& Heitzman-Powell, L. (2005). Training behavioral aides with a combination of online and face-to-face procedures. Teaching Exceptional Children, 37(5), 20-26. $10.1177 / 004005990503700503$

Cefai, J., Smith, D., \& Pushak, R. E. (2010). Parenting wisely: parent training via CD-ROM with an Australian sample. Child \& Family Behavior Therapy, 32(1), 17-33. $10.1080 / 07317100903539709$

Constantinescu, G., Theodoros, D., Russell, T., Ward, E., Wilson, S., \& Wootton, R. (2010). Assessing disordered speech and voice in Parkinson's disease: a telerehabilitation application. International Journal of language and Communication Disorders, 45(6), $630-644.10 .3109 / 13682820903470569$

Dawson, G., Rogers, S., Munson, J., Smith, M., Winter, J., Greenson, J., ... \& Varley, J. (2010). Randomized, controlled trial of an intervention for toddlers with autism: the Early Start Denver Model. Pediatrics, 125(1), e17-e23.

Deshpande, A., Khoja, S., Lorca, J., McKibbon, A., Rizo, C., Husereau, D., \& Jadad, A. R. (2009). Asynchronous telehealth: A scoping review of analytic studies. Open Medicine, 3(2), 39-61.

Fisher, W. W., Luczynski, K. C., Hood, S. A., Lesser, A. D., Machado, M. A., \& Piazza, C. C. (2014). Preliminary findings of a randomized clinical trial of a virtual training program for applied behavior analysis technicians. Research in Autism Spectrum Disorders, 8(9), 1044-1054. 10.1016/j.rasd.2014.05.002 
Fletcher, R. R., Poh, M.-Z., \& Eydgahi, H. (2010). Wearable sensors: opportunities and challenges for low-cost health care. Paper presented at the Engineering in Medicine and Biology Society (EMBC), 2010 Annual International Conference of the IEEE.

Goldstein, F., \& Myers, K. (2014). Telemental health: A new collaboration for pediatricians and child psychiatrists. Pediatric annals, 43(2), 79-84. 10.3928/00904481-2014012712

Grogan-Johnson, S., Schmidt, A. M., Schenker, J., Alvares, R., Rowan, L. E., \& Taylor, J. (2013). A Comparison of speech sound intervention delivered by telepractice and side-by-side service delivery models. Communication Disorders Quarterly, 34(4), 210-220. doi:10.1177/1525740113484965

Gros, D. F., Morland, L. A., Greene, C. J., Acierno, R., Strachan, M., Egede, L. E., . . Frueh, B. C. (2013). Delivery of evidence-based psychotherapy via video telehealth. Journal of Psychopathology and Behavioral Assessment, 35(4), 506-521. 10.1007/s10862013-9363-4

Hamad, C. D., Serna, R. W., Morrison, L., \& Fleming, R. (2010). Extending the reach of early intervention training for practitioners: A preliminary investigation of an online curriculum for teaching behavioral intervention knowledge in autism to families and service providers. Infants and Young Children, 23(3), 195.

10.1097/IYC.0b013e3181e32d5e

Harrison, E., Wilson, L., \& Onslow, M. (1999). Distance intervention for early stuttering with the Lidcombe programme. International Journal of Speech-Language Pathology, l(1), 31-36. 10.3109/14417049909167151

Hepburn, S. L., Blakeley-Smith, A., Wolff, B., \& Reaven, J. A. (2016). Telehealth delivery of cognitive-behavioral intervention to youth with autism spectrum disorder and anxiety: A pilot study. Autism, 20(2), 207-218. 10.1177/1362361315575164 
Ingersoll, B., \& Berger, N. (2015). Parent engagement with a telehealth-based parentmediated intervention program for children with autism spectrum disorders: Predictors of program use and parent outcomes. Journal of Medical Internet Research, 17(10), e227. 10.2196/jmir.4913

Ingersoll, B., Wainer, A. L., Berger, N. I., Pickard, K. E., \& Bonter, N. (2016). Comparison of a self-directed and therapist-assisted telehealth parent-mediated intervention for children with ASD: A pilot RCT. Journal of Autism and Developmental Disorders, 46(7), 2275-2284. 10.1007/s10803-016-2755-z

Jang, J., Dixon, D. R., Tarbox, J., Granpeesheh, D., Kornack, J., \& de Nocker, Y. (2012). Randomized trial of an eLearning program for training family members of children with autism in the principles and procedures of applied behavior analysis. Research in Autism Spectrum Disorders, 6(2), 852-856. 10.1016/j.rasd.2011.11.004

Keck, C. S., \& Doarn, C. R. (2014). Telehealth technology applications in speech-language pathology. Telemedicine Journal and e-Health, 20(7), 653 - 659. doi:10.1089/tmj.2013.0295.

Lewis, C., Packman, A., Onslow, M., Simpson, J. M., \& Jones, M. (2008). A phase II trial of telehealth delivery of the Lidcombe Program of Early Stuttering Intervention. American Journal Of Speech-Language Pathology, 17(2), 139-149. 10.1044/10580360(2008/014)

Lindgren, S., Wacker, D., Suess, A., Schieltz, K., Pelzel, K., Kopelman, T., .. . Waldron, D. (2016). Telehealth and autism: Treating challenging behavior at lower cost. Pediatrics, 137(2), S167-S175. 10.1542/peds.2015-28510

Lord, C., Rutter, M., DiLavore, P. C., Risi, S., Gotham, K., \& Bishop, S. (2012). Autism Diagnostic Observation Schedule, Second Edition. Torrance, CA: Western Psychological Services. 
Max, M. L., \& Burke, J. C. (1996). Virtual reality for autism communication and education, with lessons for medical training simulators. Studies in health technology and informatics, 39, 46-53.

Meadan, H., Snodgrass, M. R., Meyer, L. E., Fisher, K. W., Chung, M. Y., \& Halle, J. W. (2016). Internet-based parent-implemented intervention for young children with autism: A pilot study. Journal of Early Intervention, 38(1), 3-23. $10.1177 / 1053815116630327$

McGrath, N., Dowds, M. M., Jr., \& Goldstein, R. (2008). Clinical supervision of a client with traumatic brain injury in a host home placement using video teleconferencing: a case study. The Journal Of Head Trauma Rehabilitation, 23(6), 388-393. doi:10.1097/01.HTR.0000341434.74875.c8

National Autism Center. (2015). Findings and conclusions: National standards project, phase 2. Retrieved from: www.nationalautismcenter.org/national-standardsproject/results-reports/

O’Brian, S., Smith, K., \& Onslow, M. (2014). Webcam delivery of the Lidcombe program for early stuttering: A Phase 1 clinical trial. Journal of Speech, Language, and Hearing Research, 57, 825 - 830. doi:10.1044/2014_JSLHR-S-13-0094

Pearl, P. L., Sable, C., Evans, S., Knight, J., Cunningham, P., Lotrecchiano, G. R., . . Conway, A. (2014). International telemedicine consultations for neurodevelopmental disabilities. Telemedicine and e-Health, 20(6), 559-562. 10.1089/tmj.2013.0275

Pickard, K. E., Wainer, A. L., Bailey, K. M., \& Ingersoll, B. R. (2016). A mixed-method evaluation of the feasibility and acceptability of a telehealth-based parent-mediated intervention for children with autism spectrum disorder. Autism, 20(7), 845-855. $10.1177 / 1362361315614496$ 
Prior, M., Roberts, J. M.A., Rodger, S., Williams, K. \& Sutherland, R. (2011). A review of the research to identify the most effective models of practice in early intervention of children with autism spectrum disorders. Australian Government Department of Families, Housing, Community Services and Indigenous Affairs, Australia.

Reese, R. M., Jamison, R., Wendland, M., Fleming, K., Braun, M. J., Schuttler, J. O., \& Turek, J. (2013). Evaluating interactive videoconferencing for assessing symptoms of autism. Telemedicine and e-Health, 19(9), 671-677. 10.1089/tmj.2012.0312

Reese, R., Braun, M., Hoffmeier, S., Stickle, L., Rinner, L., Smith, C., . . Hadorn, M. (2015). Preliminary evidence for the integrated systems using telemedicine. Telemedicine and e-Health, 21(7), 581-587. 10.1089/tmj.2014.0124

Ruble, L., McGrew, J., Toland, M., Dalrymple, N., \& Jung, L. (2013). A randomized controlled trial of COMPASS web-based and face-to-face teacher coaching in autism. Journal of Consulting and Clinical Psychology, 81(3), 566-572. 10.1037/a0032003

Savin, D., Garry, M. T., Zuccaro, P., \& Novins, D. (2006). Telepsychiatry for treating rural American Indian youth. Journal of the American Academy of Child \& Adolescent Psychiatry, 45(4), 484-488. 10.1097/01.chi.0000198594.68820.59

Schutte, J. L., McCue, M. P., Parmanto, B., McGonigle, J., Handen, B., Lewis, A., . . Saptono, A. (2015). Usability and reliability of a remotely administered adult autism assessment, the autism diagnostic observation schedule (ADOS) module 4. Telemedicine and e-Health, 21(3), 176-184. 10.1089/tmj.2014.0011

Sharma, S., Ward, E. C., Burns, C., Theodoros, D., \& Russell, T. (2013). Assessing dysphagia via telerehabilitation: patient perceptions and satisfaction. International Journal of Speech-Language Pathology, 15(2), 176-183. doi:10.3109/17549507.2012.689333 
Suess, A. N., Wacker, D. P., Schwartz, J. E., Lustig, N., \& Detrick, J. (2016). Preliminary evidence on the use of telehealth in an outpatient behavior clinic. Journal of Applied Behavior Analysis, 49(3), 686-692. 10.1002/jaba.305

Sutherland, R., Trembath, D., Hodge, A., Drevensek, S., Lee, S., Silove, N., \& Roberts, J. (2017). Telehealth language assessments using consumer grade equipment in rural and urban settings: Feasible, reliable and well tolerated. Journal of Telemedicine and Telecare, 23(1), 106-115. 10.1177/1357633X15623921

Taylor, T., Webster-Stratton, C., Feil, E. G., Broadbent, B., Widdop, C. S., \& Severson, H. H. (2008). Computer-based intervention with coaching: An example using the Incredible Years program. Cognitive Behaviour Therapy, 37(4), 233-246.

\section{$10.1080 / 16506070802364511$}

Terry, M. (2009). Telemedicine and autism: Researchers and clinicians are just starting to consider telemedicine applications for the diagnosis and treatment of autism. Telemedicine and e-Health, 15(5), 416-419. 10.1089/tmj.2009.9965

Wainer, A. L., \& Ingersoll, B. R. (2013). Disseminating ASD interventions: a pilot study of a distance learning program for parents and professionals. Journal of Autism and Developmental Disorders, 43(1), 11-24. 10.1007/s10803-012-1538-4

Waite, M. C., Theodoros, D. G., Russell, T. G., \& Cahill, L. M. (2010). Internet-based telehealth assessment of language using the CELF-4. Language, Speech, and Hearing Services in Schools, 41, 445-458. 10.1044/0161-1461(2009/08-0131)

Ward, E. C., Burns, C. L., Theodoros, D. G., \& Russell, T. G. (2013). Evaluation of a clinical service model for dysphagia assessment via telerehabilitation. International Journal of Telemedicine and Applications, 2013, 918526-918526. doi:10.1155/2013/918526 
Wilson, L., Onslow, M., \& Lincoln, M. (2004). Telehealth adaptation of the Lidcombe program of early stuttering intervention: Five case studies. American Journal Of Speech-Language Pathology, 13(1), 81-93. 10.1044/1058-0360(2004/009) 